\title{
Desprofesionalizando el servicio público de asistencia a domicilio en los cuidados de larga duración: análisis de la reconfiguración del sector en la región de Madrid
}

\author{
Magdalena Díaz Gorfinkiel \\ Universidad Carlos III de Madrid \\ <mdiazg@polsoc.uc3m.es>
}

\section{Begoña Elizalde San Miguel \\ Universidad Carlos III de Madrid}

\begin{abstract}
Artikulu honen xedea da Madrilgo eskualdeko etxez etxeko arretarako sistema publikoaren azken urteotako eboluzioa aztertzea. 2008. urtetik, krisialdi ekonomikoak eragina izan du pertsona adinduen zaintzara bideratutako baliabide publikoetan, eta eragin hori nabarmendu daiteke eragina izandako arlo desberdinetan. Murriztu egin da zerbitzuaren intentsitatea eta baita ere mendetasun egoeran dauden pertsonei eskainitako beste zerbitzu batzuen erlazioan izandako garrantzia, eta, gehienbat, jarduera horiek betetzen dituzten pertsonen lanerako baldintzak eskastu dira. Gaur egun, murriztu egin da jardueren inguruko kontrola, eta horren ondorioz izaera profesionaleko ezaugarri batzuek eskastu egin dira mendetasun egoeran dauden pertsonen arretan, eta jarduera horiek aintzatespen eta zehaztasun gutxiagoa duten etxebizitzako mantentzearen lanen tankera bat hartu dute. Halaber, prestakuntzarako ikastaroak gutxitu egin dira langileentzat, eta nabarmentzen da sektorean esperientzia urria duten enpresak protagonista bilakatzen ari direla baliabide horiek eskaintzeko.
\end{abstract}

\section{GAKO-HITZAK:}

Etxez etxeko arreta, izaera profesionalaren atzerakada, lanerako baldintzak, krisialdi ekonomikoa.
El presente artículo tiene como objeto analizar la evolución del sistema público de asistencia a domicilio en la región de Madrid en los últimos años. Desde 2008, la crisis económica ha afectado a los recursos públicos destinados al cuidado de mayores, lo que se puede percibir en el funcionamiento de este recurso en diferentes ámbitos. Se ha recortado la intensidad del servicio y su importancia en relación con otros servicios ofrecidos para la atención de personas dependientes, $y$, fundamentalmente, se han deteriorado las condiciones de trabajo de las personas que realizan estas tareas. Existe en la actualidad un menor control de las actividades que se realizan, motivo por el que el trabajo de cuidados a personas dependientes ha perdido los rasgos de profesionalización, para asemejarse a un empleo de mantenimiento del hogar con escaso reconocimiento y concreción de tareas. Además, se está asistiendo a la disminución de cursos de formación y reciclaje de trabajadoras, y se está permitiendo que compañías carentes de experiencia en el sector adquieran protagonismo en la prestación de este recurso.

\section{Palabras Clave:}

Asistencia a domicilio, desprofesionalización, condiciones de trabajo, crisis económica. 


\section{Introducción}

Los cuidados de la población mayor constituyen un ámbito de actuación fundamental en la sociedad española actual. Esto se debe al envejecimiento de la población que ha experimentado este país durante las últimas décadas. El Censo de Población y Vivienda de 2011 (INE, 2011) indica que las personas mayores de 65 años representan el $17,3 \%$ de la población, lo que en números absolutos significa más de 8 millones de personas.

El envejecimiento demográfico en España presenta dos características. En primer lugar, a pesar de iniciarse más tarde que en otros países de su entorno ha sido un proceso acusado e intenso (Imserso, 2012); así, el porcentaje de mayores de 65 años ha aumentado 0,7 puntos porcentuales en apenas dos años (en 2009, representaban el 16,6\%). El otro rasgo peculiar del envejecimiento poblacional en el país es el llamado sobreenvejecimiento, es decir, la elevada proporción de personas mayores de 80 años (5,3\% según datos del INE), siendo España uno de los países con mayor esperanza de vida de todo el mundo. El envejecimiento dentro de la propia población mayor ocasiona importantes necesidades de cuidado con la consecuente obligación de generar recursos para dar respuesta a esta demanda, en función de la relación que existe entre la edad y las situaciones de discapacidad. La situación demográfica actual plantea, en consecuencia, el reto de la calidad de vida durante los años en que ésta se ha extendido (Bódalo Lozano, 2006) así como un desafío para los gobernantes y el desarrollo de la políticas sociales (Minguela Recover y Camacho Ballesta, 2015).

En las sociedades contemporáneas la atención a las personas mayores en situación de dependencia constituye un derecho fundamental (De Asís Roig, 2007), por lo que el Estado, las familias y el mercado se complementan con objeto de que esta necesidad de cuidados se vea atendida. España se ha caracterizado tradicionalmente por un Estado de bienestar de carácter familista, una característica compartida con otros países mediterráneos que implica que gran parte del desarrollo del bienestar social, y en este caso concreto de la responsabilidad de los cuidados, recae en el grupo familiar. La estructura familiar, sin embargo, se ha transformado en los últimos años conforme a las transformaciones sociales experimentadas en el país, haciendo que los 'viejos' modelos familiares sólo pervivan en apariencia (Moreno Fernández, 2003). Las familias se han visto sujetas a procesos de cambio, entre otras cosas, por sus bajas tasas de fecundidad y el nuevo papel de la mujer en la sociedad. El aumento de la participación femenina en el mercado de trabajo no se ha visto acompañada por un aumento de participación masculina en las tareas de cuidado (INE, 2009-2010). Esto ha provocado que la forma tradicional de cuidar, centrada en la familia, haya quedado obsoleta, un proceso que ha sido conceptualizado como "crisis de los cuidado"
(Hochschild, 2001) y que hace referencia a la menor disponibilidad de las familias para cuidar y la consecuente necesidad de emplear otras estrategias.

La institucionalización de los cuidados ha ido así adquiriendo mayor protagonismo social y ha generado la aparición de relaciones formales dentro de la provisión de los cuidados a la dependencia (Fantova Azcoaga, 2013). Sin embargo, Moreno Colom et al. (2013) señalan que a pesar de estos cambios en la organización social de los cuidados aún se presentan resistencias socioculturales hacia los cuidados colectivos y la presencia de los servicios sociales, otorgándosele preferencia al trabajo informal en el seno de la familia. De esta forma, la originaria escasez de servicios de cuidados propia del Estado de bienestar español se ve justificada y, al mismo tiempo, estimulada por la tendencia social a satisfacer este tipo de actividades en el ámbito de lo privado.

La formalización pública de los servicios de cuidados a la población dependiente, y en consecuencia a gran parte de la población mayor en su conjunto, experimentó un enorme avance con la aprobación de la Ley de Promoción de la Autonomía Personal y Atención a las Persona en Situación de Dependencia en 2006 (Ley 39/2006, conocida como Ley de Dependencia). Esta Ley significó la asunción por parte del Estado de la responsabilidad en el bienestar de la población dependiente, con la consecuente conformación de los cuidados como un campo de ciudadanía y no discriminación. Una de las primeras consecuencias de su aprobación es el aumento en el gasto público destinado a los cuidados, a pesar de lo cual España está aún lejos de las cifras de los países europeos con mayor inversión. En 2003 el porcentaje del PIB destinado a este tipo de cuidados era de $0,77 \%$, cifra que aumentó hasta $1,11 \%$ en 2010 , pero que contrasta con el $3,71 \%$ de los Países Bajos o el 3,70\% de Suecia (Imserso, 2012).

La Ley de Dependencia, desde sus inicios, se planificó con un desarrollo progresivo que permitiese proporcionar los servicios técnicos, asistenciales y de prestación económica necesarios en función de la complejidad de la tarea y estructuras demandadas por las personas dependientes (González Ortega, 2012)1. Desde la aprobación de la Ley, se estableció un catálogo de servicios (institucionales y a domicilio) y unas prestaciones económicas (ayudas monetarias directas) que se adjudicarían en función del grado de dependencia de las personas, sus recursos económicos y las posibilidades reales de los servicios sociales en cada región, dando prioridad a la prestación de servicios (González Ortega, 2012). Entre los servicios que se planificaron, y que se ofrecen en la actualidad, se encuentra el servicio de ayuda a domicilio, servicio que se encontraba muy desarrollado con anterioridad a la Ley de Dependencia y que no ha experimentado el desarrollo que cabría esperar, al haber dado la Ley

${ }^{1}$ El desarrollo de la Ley experimentó postergaciones respecto al calendario inicial. 
prioridad a las prestaciones económicas entrando en contradicción con su espíritu inicial. Este tipo de prestaciones representaban, en diciembre de 2014, el 48,37\% del total de prestaciones otorgadas en España, mientras que la ayuda a domicilio representaba el 14,26\% (Imserso, Sistema de Autonomía y Financiación a la Dependencia).

Este artículo se centra en el análisis del servicio público de asistencia a domicilio de los cuidados de larga duración en la región de Madrid. El objetivo es examinar la evolución que ha experimentado este servicio en la región, coincidiendo con la implantación de la Ley de Dependencia y el desarrollo de la crisis económica en España. Este análisis forma parte de un proyecto de investigación más amplio que estudia de manera comparativa y a escala regional la organización social de los cuidados de mayores en España ${ }^{2}$, observando la interconexión entre los recursos proporcionados por el Estado, la familia y el mercado. El artículo comienza presentando el diseño de la investigación, para luego centrarse en sus secciones principales: el análisis de la evolución del recurso de la ayuda a domicilio en la Comunidad de Madrid y los elementos presentes en la reconfiguración de esa prestación.

\section{Diseño de la investigación}

El proyecto se apoya en una estrategia de triangulación metodológica que combina la explotación de fuentes cuantitativas y cualitativas. En este caso concreto, se presentan algunos de los datos obtenidos en el desarrollo de la investigación de la Comunidad de Madrid, investigación que aún se encuentra en proceso de realización y análisis. Acorde con los objetivos del artículo, en este caso se ofrecerán exclusivamente datos relativos a la situación del servicio de atención a domicilio, relegando la información del resto de ámbitos para posteriores análisis.

En primer lugar se utilizan datos cuantitativos secundarios con objeto de observar la evolución de este servicio en la región de Madrid y comprender el papel que se le otorga dentro de las prestaciones a la población dependiente. El servicio de ayuda a domicilio (SAD) se estudia realizando un seguimiento de su evolución en la región, aludiendo a la cobertura que ofrece tanto en términos absolutos como en comparación con otras comunidades autónomas así como en la importancia de la prestación en relación con otras prestaciones. Todos los datos provienen de informes que el Imserso realiza de manera periódica y que se constituyen como un reflejo de la forma de entender este servicio tanto desde una perspectiva de actuación práctica como desde un punto de vista simbólico.

2 Proyecto 'Geografía del trabajo de cuidados. Implicaciones de su privatización sobre la creación de empleo' (CSO2012-32901), dirigido por Raquel Martínez Buján y financiado por el Ministerio de Empleo y Competitividad.
El análisis de las transformaciones en la cotidianeidad del funcionamiento del SAD se obtiene, por su parte, con la información que proporciona el trabajo de campo realizado en la región de Madrid. Se planificaron, al igual que en el resto de regiones a estudiar, 30 entrevistas con objeto de cubrir las tres aristas del cuidado: el Estado, la familia y el mercado. Se seleccionaron, en consonancia, diferentes perfiles para entrevistar, con objeto de obtener información que complemente e interconecte la actuación de los actores sociales que participan en la provisión de cuidados de larga duración. Para este artículo se utilizan, fundamentalmente, las entrevistas realizadas a las trabajadoras del SAD y a los gestores y técnicos de los servicios públicos. En relación con las primeras, se realizaron dos entrevistas a trabajadoras del SAD que en ambos casos eran trabajadoras de origen inmigrante que se encontraban contratadas por empresas concertadas y realizaban su actividad en el municipio de Madrid. Ambas llevaban más de cinco años trabajando en el sector y provenían del ámbito del empleo del hogar. Para el segundo grupo, por su parte, se entrevistó a tres coordinadoras de los servicios de atención a domicilio, trabajando dos de ellas para el Ayuntamiento de Coslada y la tercera para una empresa de servicios sociosanitarios que ofrece atención a domicilio de manera concertada en municipios del norte de Madrid (como Las Rozas o Majadahonda). Las dos entrevistas realizadas a los cuidadores familiares y las siete a los cuidadores profesionales empleados del hogar se constituyen como un complemento de información que permite una visión global de la evolución de la organización social de los cuidados a la población dependiente. Las entrevistas fueron realizadas durante 2014 y siguieron un guión semiestructurado, que permitió incluir los temas que se pretendían analizar, permitiendo la flexibilidad en el desarrollo de los discursos de los entrevistados. Para el caso de las trabajadoras del SAD, los bloques que se incluyeron se referían a las características del empleo, la trayectoria laboral (dentro del sector y previamente a la inserción en él), la formación específica para la realización de este trabajo, las relaciones que se establecen en el desarrollo de la actividad y, finalmente, la percepción de los cuidados propios y ajenos. Para el caso de las gestoras del SAD, el guión incluía bloques referidos a las características del trabajo en concreto, las formas de implementación de la Ley de Dependencia, el impacto de la Ley en el sector de los cuidados, los cambios acaecidos en los últimos años de crisis económica y una valoración del proceso de prestación de cuidados de larga duración.

\section{Recursos públicos en la Comunidad de Madrid: el servicio de atención a domicilio}

La Ley de Dependencia es una ley de ámbito estatal que tiene por objeto garantizar las condiciones básicas de provisión de cuidados a las situaciones de dependencia y que se ha desarrollado delegando su gestión e implantación a las comunidades autónomas 
(González Ortega, 2012). Este diseño ha originado la existencia de una gran variedad de niveles de desarrollo regional (Martínez Buján, 2014), que no ha hecho más que consolidar las diferencias previamente existentes, cuando las prestaciones se ofrecían fuera de este marco normativo. La Ley establece un catálogo de servicios donde se especifican las prestaciones existentes, y entre ellas, se establece el servicio de atención a domicilio (SAD). Éste se define como "el conjunto de actuaciones llevadas a cabo en el domicilio de las personas en situación de dependencia con el fin de atender sus necesidades de la vida diaria” (Ley de Dependencia, artículo 23), lo que implica un esfuerzo por prestar la atención necesaria dentro del entorno habitual de la persona dependiente, siguiendo la línea de expertos y organismos internacionales que defienden esta opción como preferente (García Martín, 2013). Este tipo de prestación puede asumir dos tipos de asistencia: la relacionada con la atención personal y la relacionada con la atención de las necesidades domésticas o del hogar. Los servicios de atención a domicilio son gestionados por la Administración pública, pero pueden ser prestados tanto por entidades públicas como por empresas privadas, previa adjudicación del servicio por parte de la Administración pública.

En el caso concreto de la Comunidad de Madrid, la ayuda a domicilio se rige por varios decretos que conviven y combinan realidades territoriales de diverso nivel. El Decreto 88/2002 regula este tipo de prestaciones a escala autonómica y local, y es previo al desarrollo de la Ley de Dependencia. En él, se establece la importancia que el Gobierno de la región otorga a este tipo de servicios de asistencia al establecer que "la ayuda a domicilio [es] una de las más relevantes prestaciones del sistema de servicios sociales que, a lo largo de estos últimos años, ha experimentado un importante crecimiento, incrementándose los recursos humanos y financieros disponibles, los niveles de cobertura y las modalidades de prestación del servicio ofertadas a la población, gracias al esfuerzo conjunto de las administraciones autonómica y local”. El funcionamiento autonómico y municipal, previo a la Ley de Dependencia, generaba una cobertura individualizada y específica en cada región (García Herrero, 2009), siendo la Comunidad de Madrid una de las regiones que más habían desarrollado este tipo de servicios (Martínez Buján, 2014). Dentro de las modalidades de ayuda a domicilio, el Decreto estipula la atención doméstica y la atención personal de la misma manera que la Ley de Dependencia, sin establecer una relación de complementariedad entre ellas. La aprobación de la Ley de Dependencia significó el desarrollo de decretos y resoluciones estatales y regionales con objeto de lograr una acertada puesta en práctica de la Ley. ${ }^{3}$ En 2013 , se

3 Entre ellos, el Decreto 54/2015, que regula el procedimiento para reconocer la situación de dependencia y el derecho a las prestaciones en la Comunidad de Madrid; el Real Decreto 291/2015, que modifica las prestaciones del sistema aprobadas en 2013; o la Resolución $8 / 2015$, que regula el catálogo de servicios y prestaciones para las personas reconocidas en situación de dependencia en grado I en aprueba el Real Decreto 1051/2013, que ordena y unifica la normativa relacionada con las prestaciones y servicios. Lo más relevante de este real decreto, en lo que respecta al análisis del SAD, es la incorporación de la complementariedad entre la ayuda personal y doméstica, así como el establecimiento de una nueva intensidad en la percepción del servicio. En cuanto a la primera cuestión, se establece que la asistencia doméstica sólo podrá prestarse, a no ser en casos excepcionales, como complementario de la asistencia personal y que dentro del programa de atención de cada individuo se deberán especificar las horas otorgadas a cada servicio. En cuanto a la intensidad de los servicios, se establecen niveles que son inferiores a los establecidos en las normativas anteriores, lo que indica una tendencia a la disminución formal en la oferta de éste. Las cifras establecidas en la actualidad son las siguientes:

- Grado III, gran dependencia: entre 46 y 70 horas mensuales.

- Grado II, dependencia severa: entre 21 y 45 horas mensuales.

- Grado I, dependencia moderada: máximo de 20 horas mensuales.

Previamente a la implantación de esta normativa las horas máximas de prestación de atención a domicilio eran 90 para el grado III de dependencia, 55 para el grado II y 30 para el grado I, teniendo en cuenta además que dentro de cada grado se establecían diferentes niveles. La entrada en vigor del citado real decreto ha producido una reducción de la intensidad del servicio ofertado así como una simplificación en su asignación debido a que ya no existen niveles en cada grado y, como consecuencia, una potencial necesidad de hacer frente a la situación de dependencia con recursos complementarios a la atención pública a domicilio.

En el caso de la Comunidad de Madrid, no sólo los datos estatales provenientes de la normativa indican una disminución en la prestación de este servicio, sino que también las cifras referidas a la cobertura del SAD reflejan una tendencia a la baja en los últimos años ${ }^{4}$. La Tabla 1 presenta los datos relativos al número de personas atendidas por este servicio, el porcentaje de personas beneficiarias en relación con los beneficiarios de todos los servicios, el porcentaje de personas beneficiarias respecto a la población mayor de 65 años, la prestación más representativa en la región en cada año (es decir, la que tiene una proporción de beneficiarios mayor) y la posición que este servicio representa en relación con a otras regiones (es decir, cuántas regiones del país tienen un porcentaje de beneficiarios mayor).

la Comunidad de Madrid.

${ }_{4}^{4}$ A pesar de que la Ley de Dependencia armonizó a nivel estatal los servicios prestados a las personas en situación de dependencia, los Ayuntamientos mantienen prerrogativas sobre este tipo de servicios y, en consecuencia, pueden ofrecer atención a domicilio en los casos específicos que lo consideren necesario. 
Tabla 1. Evolución del SAD bajo la Ley de Dependencia. Comunidad de Madrid, 2010-2015*

\begin{tabular}{|l|c|c|c|c|c|}
\cline { 2 - 6 } \multicolumn{1}{c|}{} & No personas & $\begin{array}{c}\text { \% beneficiarios de } \\
\text { todas las prestaciones }\end{array}$ & $\begin{array}{c}\text { \% beneficiarios SAD } \\
\text { entre la población > } \\
65 \text { años** }\end{array}$ & $\begin{array}{c}\text { Prestación más } \\
\text { representativa }\end{array}$ & $\begin{array}{c}\text { Posición en relación } \\
\text { con otras regiones }\end{array}$ \\
\hline 2010 & 7.292 & 13,16 & 0,74 & $\begin{array}{c}\text { Atención residencial } \\
(34,55 \%)\end{array}$ & $4^{\underline{a}}$ \\
\hline 2011 & 21.593 & 22,15 & 2,16 & SAD & $1^{\underline{a}}$ \\
\hline 2012 & 23.156 & 23,01 & 2,26 & SAD & $1^{\underline{a}}$ \\
\hline 2013 & 21.356 & 19,37 & 2,03 & PECEF (26,96\%) & $4^{\underline{a}}$ \\
\hline 2014 & 19.595 & 17,20 & 1,81 & PECEF (25,40\%) & $6^{\underline{a}}$ \\
\hline 2015 & 19.829 & 17,61 & 1,84 & PECEF (24,70\%) & $6^{\underline{a}}$ \\
\hline
\end{tabular}

SAD: servicios de asistencia domiciliaria. PECEF: prestación económica para el cuidado en el entorno familiar.

* Datos a 31 de diciembre, salvo los de 2015, que son a 30 de junio.

** A partir de los datos poblacionales a 1 de enero de cada año.

Fuente: información estadística del Sistema de Autonomía y Atención a la Dependencia (Imserso, 2010-2015), y padrón continuo (INE).

Hasta finales de 2012 la presencia del SAD fue aumentado en relación con a otras prestaciones, un dato coherente con el importante esfuerzo que se realizó en las mayor parte de las regiones de España para aumentar su presencia y potenciar su uso (Martínez Buján, 2014). En estos primeros años, las cifras oscilan en torno a un $22 \%$, y constituía la prestación más frecuente en la Comunidad de Madrid, por encima del resto de servicios y prestaciones. Asimismo, se ve que durante esos años esta región se posicionaba en primer lugar en la oferta de este servicio en el conjunto del país. Estos datos, sin embargo, no deben esconder la disparidad existente respecto a la intensidad en la oferta del servicio. La Comunidad de Madrid, por ejemplo, presenta una intensidad horaria de 16,98 horas al mes por usuario (Imserso, 2012) lo que es inferior a otras regiones (encontrándose en el puesto 11을 es decir, que existen diez regiones que presentan una intensidad horaria mayor). Estas diferencias son indicativas de la existencia de estrategias variadas a escala regional y municipal (Martínez Buján, 2014) respecto a si priorizar la cantidad de población que recibe el servicio o la intensidad horaria de disfrute de las prestaciones. Los datos también muestran otra diferencia en relación con la distribución de modalidades dentro del SAD, es decir el equilibrio entre las tareas destinadas a cuidados personales y las centradas en la ayuda doméstica. En Madrid, en 2011 , el $66 \%$ del tiempo se dedicaba a la primera tipología, mientras que el $34 \%$ restante se concentraba en la ayuda en el hogar (Imserso, 2012), unas cifras que, sin embargo, deben contrastarse con los discursos de los actores sociales involucrados respecto a las prácticas reales de esta distribución.

Volviendo a la evolución durante los últimos años, se puede observar a partir de 2013 un claro descenso en la importancia del SAD respecto a las demás prestaciones, a pesar de mantener cifras elevadas tanto en los números absolutos de cobertura como en el peso del servicio en relación con otros. El primer indicador de su tendencia a la baja se puede observar en la disminución de beneficiarios respecto a la población mayor de 65 años, dato que debe analizarse desde dos puntos de vista. Por un lado, desciende el número absoluto de personas atendidas (entre 2012 y 2013 el número de beneficiarios descendió un $8 \%)^{5}$. Pero además, y teniendo en cuenta el constante aumento de la población mayor de 65 años debido al envejecimiento demográfico, descendió de forma muy acusada (un $15 \%$ ) la cobertura a la población mayor, es decir, el porcentaje de mayores que acceden a este servicio.

Esta disminución de la oferta en el SAD, además, estuvo acompañada por un constante aumento en las prestaciones económicas para cuidados familiares desde 2010. Se percibe una correlación directa entre el importante descenso del SAD entre 2012 y 2013 y un aumento en ese mismo periodo de estas prestaciones (de representar un 19,14\%

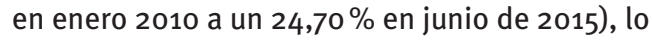
que parecería indicar una menor apuesta respecto a la profesionalización de estos cuidados frente a una progresiva priorización de la presencia familiar y sus cuidados no formalizados. De hecho, desde 2013 estas prestaciones económicas han pasado en Madrid a ser la prestación más habitual. Asimismo, otro tipo de prestación económica, la vinculada al servicio, ha ido aumentando progresivamente su importancia desde 2010, produciéndose un salto notable entre 2012 y 2013 , pasando de significar un $4,97 \%$ a un $8,61 \%$ (Imserso, Sistema de Autonomía y Atención a la Dependencia). Esta prestación tiene como objetivo cubrir los gastos de un servicio de atención cuando éste no pueda ser proporcionado directamente por las administraciones públicas e indica, nuevamente, una tendencia a la externalización de la atención a domicilio.

Otro elemento que puede ser indicativo de la disminución en la profesionalización o importancia otorgada a este ámbito de prestaciones es el

${ }^{5}$ Los datos ofrecidos se refieren a toda la población beneficiaria del SAD dentro del marco de la Ley de Dependencia, ya que no se han facilitado datos desagregados por edades, pero se debe tener en cuenta que el $65 \%$ de los beneficiarios del SAD en la Comunidad de Madrid son mayores de 80 años (Imserso, 2102) y el 75,85\% de los solicitantes de las prestaciones en su conjunto para todo el territorio nacional son mayores de 65 años (Imserso, para septiembre de 2015). 
relativo a las empresas adjudicatarias del servicio, ya que se percibe en la región una tendencia a la externalización indiscriminada (García Martín, 2013). En el municipio de Madrid se otorgó el servicio, desde el 1 de diciembre de 2012 hasta el 31 de marzo de 2015, a las empresas Clece, Asispa y Eulen. Clece, por ejemplo, es filial de una empresa constructora que ofrece multiservicios sin haber estado especializada en la atención a domicilio ni constituir éste su ámbito preferente de actuación. Dentro del debate de la adjudicación de estas prestaciones se señala que estas grandes corporaciones, suelen hacerse con los servicios a través de una mejor propuesta de precios, lo cual sólo estas empresas pueden realizar en función de su mayor tamaño y su capacidad para gestionar grandes lotes de servicios. Además, se plantea la duda de si estos menores precios ofrecidos a la Administración no se encuentran relacionados con peores condiciones laborales y una menor profesionalización de los trabajadores. Unos rasgos que caracterizan no sólo la externalización de los servicios de la Ley de Dependencia, sino, en general, la privatización del sector de la salud en España (Eurofound, 2015). La adjudicación a este tipo de empresas de la gestión de los servicios públicos incluidos en la Ley de Dependencia ha hecho plantearse la cuestión de la ideología que subyace a este diseño en la estructura y gestión de los recursos públicos, y las consecuencias que tiene la entrada de estas empresas no especializadas en la desvalorización del SAD, cuestión que pasamos a analizar a continuación.

\section{Reconfiguración del paradigma de la atención a domicilio: el discurso de la desprofesionalización}

Además de los datos que proporcionan información sobre los cambios acaecidos en el ámbito de la ayuda a domicilio, las entrevistas realizadas ofrecen información complementaria respecto a las modificaciones experimentadas y permiten contrastar algunos de los 'datos oficiales'. Las entrevistadas ofrecen detalles sobre el funcionamiento de cuestiones cotidianas de la organización del trabajo de ayuda a domicilio, y en sus discursos dan cuenta de las restricciones que se han ido generando y las mayores dificultades que encuentran en los últimos años para ofrecer un servicio de calidad. La mayor parte de los elementos que se señalan no aluden específicamente a una cuestión de cobertura, intensidad o indicador similar, sino que proporcionan información sobre elementos concretos que conforman el servicio y que construyen en su conjunto un determinado tipo de atención a domicilio.

Comenzando por la organización de los horarios laborales la involución sufrida se presenta de manera clara al haberse planteado limitaciones para la gestión del tiempo. El principal cambio que se menciona alude a la eliminación de los tiempos de descanso entre distintos usuarios, por lo que el tiempo de traslado entre los diferentes hogares debe descontarse del tiempo de trabajo de las empleadas ${ }^{6}$. Así, si el tiempo de traslado de una localización a otra es de quince minutos, serán también quince los minutos que la empleada deba añadir a su jornada. Esto convierte el horario pactado en un contrato ficticio, cuyo cumplimiento se presenta imposible, puesto que ambas partes saben que no responde a la realidad, lo que genera descontento y presión en los trabajadores. Este tipo de funcionamiento, además de la aceptación formal de unas condiciones irreales, añade incertidumbre a las jornadas laborales ya que éstas dependerán de la localización de los usuarios y no de la capacidad de trabajo de las empleadas o de su negociación con la empresa o entidad. La modificación de la residencia de un usuario o cualquier cambio similar podría implicar también la modificación de la jornada laboral. Por otra parte, la eliminación de los tiempos de descanso trae consigo otra importante consecuencia para la relación laboral: la desaparición de los tiempos de reunión. Se señala que los tiempos de descanso que existían previamente se utilizaban para realizar traslados o para reunirse con otras empleadas cercanas. Estos encuentros posibilitaban el intercambio de información relativo a formas de trabajo y a las condiciones de éste. Su ausencia significa la imposibilidad de transmitir experiencias que permitan innovar o mejorar las formas de trabajar y de relacionarse con los usuarios, y en este sentido se podría considerar como una pérdida para el servicio y las empresas que lo gestionan. Esta restricción para compartir la realidad laboral podría interpretarse, asimismo, como una forma de control de los trabajadores ya que de esta manera los empleados desconocen las condiciones de trabajo imperantes en el sector y, en consecuencia, no generan demandas colectivas al respecto. La atomización de los trabajadores se ha demostrado como una técnica de desactivación de demandas laborales que podría presentarse como deseable en un contexto de recortes de derechos (las empleadas del SAD señalaban, precisamente, que abandonaron el sector del empleo doméstico para insertarse en éste, debido a las mejoras condiciones laborales que presentaba y al hecho de poder conocerlas):

Este contrato permite que no tenga descanso entre usuarios, por lo que lo que tardo en ir de una casa a otra lo saco de mi propio tiempo, con lo cual siempre acabo más tarde... (Romina, empleada del SAD, Madrid, 2014)

En cuanto a los cambios en la jornada laboral, también se apuntan dos muestras del empeoramiento de las condiciones. La primera hace referencia a la consideración de las horas extras, las cuales presentan una desmejora general en su funcionamiento, a pesar de que existe cierta

${ }^{6}$ Se utilizará el término femenino, por ser las mujeres la mayor parte de trabajadoras del sector. 
variabilidad entre las empresas encargadas de gestionar el servicio. En algunos casos, las horas extras han dejado de abonarse, mientras que en otras ocasiones se pagan, pero son decididas de manera unilateral por la empresa, coincidiendo normalmente las solicitudes con los fines de semana. La segunda cuestión mencionada se refiere a la recuperación de las horas de trabajo perdidas por causas ajenas a los trabajadores. En algunas ocasiones los usuarios deben trasladarse al médico o no se encuentran en su casa por decisión personal $y$, por tanto, las empleadas no deben asistir a estos hogares. Cuando esto ocurre, las empleadas deben recuperar las horas de servicio no proporcionadas, por lo que las enfermedades de los usuarios terminan repercutiendo negativamente en sus formas de trabajo. Dentro de esta dinámica, además, se debe tener en cuenta que en la población mayor dependiente la enfermedad no constituye un hecho aislado, sino una realidad recurrente, de manera que las adaptaciones horarias de las empleadas se conforman como una cuestión habitual.

[Los fines de semana, ] para recuperar horas que me faltan [...], si me falta algún... día, se me pone mal alguna persona o lo llevan al hospital, pues recupero horas. El sábado por la mañana hago tres horas y media... (Verónica, empleada del SAD, Madrid, 2014)

Por otro lado, es necesario señalar una cuestión que se apunta de forma indirecta en el discurso de las entrevistadas y es la referida a la relación entre la forma de prestar el servicio y número de horas de prestación. El Real Decreto 1051/2013 redujo el número de horas máximas a percibir por parte de los usuarios, por lo que las necesidades de una persona deben verse respondidas en la actualidad en una menor cantidad de tiempo, afectando esto a la relación entre las partes y la satisfacción con el servicio.

En cuanto a las tareas que se incluyen dentro de la prestación de los servicios, las entrevistadas también señalan una notoria desregulación, que repercute negativamente en su condición como trabajadoras. En los discursos se señala que progresivamente se ha generado una indefinición en la realización de tareas que ha llevado a que no existan límites claros a lo que se les pueda demandar, confundiendo las modalidades de la Ley (de cuidados y ayuda en el hogar) y excediendo la propia normativa para cada una de ellas. Señalan que antiguamente las tareas realizables se encontraban bien delimitadas y que las que se solicitasen fuera de norma se podían rechazar, pero que en el momento actual las empresas gestoras exigen flexibilidad en la aceptación de dichas tareas. Se les pide explícitamente que acepten 'lo que haya', incluso cuestiones como limpiar alfombras, que exceden los servicios de ayuda a domicilio. Consideran que este cambio se ha producido de manera progresiva desde los inicios de la crisis económica, pero sin una directiva explícita al respecto, por lo que hace más difícil oponerse. Se empiezan a confundir, por tanto, las tareas relacionadas con la profesión con aquellas a realizar como favor y, consecuentemente, la capacidad de las empleadas de hacer valer su profesión se ve disminuida. En esta misma línea, se señala que los servicios solicitados se relacionan cada vez más con la prestación de tareas del hogar de manera independiente a la atención de las personas, traicionando de esta manera el espíritu de la propia Ley.

$$
\begin{aligned}
& \text { Aunque ahora, con el... trabajo y eso, pues..., } \\
& \text { mm..., la coordinadora nos dice: “lo que } \\
& \text { haya” [...], porque está muy mal la cosa. Antes } \\
& \text { teníamos..., eh..., mm..., ¿cómo te digo?..., un } \\
& \text { tope de cosas. No podíamos subirnos hasta una } \\
& \text { escalera muy alta, ni a sitios muy altos...; tampoco } \\
& \text { podíamos limpiar este..., ¿cómo se llaman?, } \\
& \text { las alfombras, eso..., o sea, arrodillarnos..., no. } \\
& \text { (Verónica, empleada del SAD, Madrid, 2014) }
\end{aligned}
$$

Por último las entrevistadas aluden a la presencia de empresas gestoras y la relación con ellas. Lo primero que se señala es la variedad de empresas que prestan servicios y la diversidad de condiciones que cada una de ellas establece. Además se alude a los cambios recientes en la presencia de empresas gestoras y a la falta de relación de éstas con los antiguos proveedores de los servicios (fundamentalmente ayuntamientos).

$$
\begin{aligned}
& \text { Desde el año pasado, desde abril del año pasado, } \\
& \text { ya es una nueva empresa que lo ha adscrito la } \\
& \text { Comunidad de Madrid, con lo cual todos esos } \\
& \text { servicios..., ya como que nos lo ha quitado a } \\
& \text { los ayuntamientos..., nos lo[s] han quitado, } \\
& \text { entre comillas. La gestión de las horas y tal } \\
& \text { es a través de una empresa; el precio hora lo } \\
& \text { ha dispuesto también la Comunidad [...]. Muy } \\
& \text { aisladas, porque antes de que saliera la Ley de } \\
& \text { Dependencia, nosotros trabajábamos con todo } \\
& \text { lo que era centros de día, gestión de centros } \\
& \text { de día, gestión de residencias, y tal, y siempre } \\
& \text { teníamos algún teléfono de referencia. Entonces } \\
& \text { siempre había alguna compañera que estaba al } \\
& \text { otro lado y donde te orientaba y te decía... [...]. Es } \\
& \text { muy difícil contactar y hablar con ellos, de hecho, } \\
& \text { no tenemos ningún tipo de teléfono... (María, } \\
& \text { trabajadora social, Coslada, 2014) }
\end{aligned}
$$

Entre la disparidad de condiciones que se encuentran las empleadas según la empresa a la que pertenezcan, además de las previamente mencionadas respecto a las horas extras, se apuntan los distintos salarios y las diferentes formas de afrontar las bajas por enfermedad. Los salarios los establece la empresa gestora independientemente del precio que se cobra por el servicio, y éstos oscilan entre los 700 euros por 36 horas de trabajo hasta los 850 por 40 horas, aunque no se señala que se hayan visto modificado en los últimos años (el ajuste salarial se ha realizado mediante el control del tiempo de trabajo ya mencionado). En cuanto a las bajas por enfermedad, en algunas empresas se respetan, tal como establece 
la Ley, mientras que en otras se conceden únicamente en situaciones muy graves (habiéndose dado el caso de contagio a los usuarios - de conjuntivitis en este caso- por haber tenido que cumplir la jornada laboral en condiciones no adecuadas), cuestión que empezó a acontecer en los últimos años. La divergencia respecto a las condiciones en los distintos ámbitos refleja que la delegación en empresas distintas, y que han ido cambiando, implica una falta de concreción en la profesión de las empleadas del SAD así como la idea de que no existen unas características y cualificaciones concretas, sino que se establecen a la medida del contexto socioeconómico y de primacía empresarial.

Otro de los cambios que se señalan en relación con el mantenimiento de la calidad del servicio es el relativo a la escasez de los cursos de formación. Se apunta que antiguamente se ofrecían numerosos cursos de este tipo (aunque fuera del horario laboral) con objeto de mejorar técnicas específicas de cuidado de dependientes, pero que en la actualidad se ofrecen en mucha menor cantidad. Se confirma así la falta de un SAD especializado (García Herrero, 2009) ya que no se proporciona formación específica para situaciones concretas de dependencia (como alzhéimer) lo que repercute en la forma de prestar y recibir el servicio. También se señala una disminución en la profesionalidad ofrecida en cuanto a la información que las trabajadoras obtienen previamente a la prestación de un servicio. Esto también varía en función de la empresa gestora, pero se apunta el hecho de que en ocasiones se asiste a usuarios sobre quienes no se ha obtenido información previa de su situación. Esto ocurre tanto para la suplencia en hogares como para el acompañamiento a hospitales de nuevos usuarios.

Porque ahora están llamando, ¿sabes? Están llamando, pero... yo no sé, creo que sólo a ciertas compañeras. [...] Sí, sí, sí [te sirven los cursos], cómo movilizarlo, eso es muy importante..., porque muchas personas, o sea, ¿qué te digo...?, señoras, vienen los hijos y "si es que tú no puedes, porque estás muy delgada”. No es que... por estar delgada, sino cómo debemos de levantar a las personas, moverlos, para no hacernos daño ni hacerles daño a ellos. (Verónica, empleada del SAD, Madrid, 2014)

En definitiva, el conjunto de cambios identificados por los agentes sociales revelan que las modificaciones concretas que han tenido lugar en los últimos años, en un contexto político de recortes y austeridad, están repercutiendo en la construcción de un sector desprofesionalizado donde los servicios a prestar se conforman como un conjunto difuso de tareas o cualificaciones sin requerimientos claros. Todos los cambios indican esta tendencia hacia la reconfiguración del paradigma de la ayuda a domicilio, asentando la ya preexistente idea del ámbito del cuidado de personas mayores como un sector sin una profesión específica (Moreno Colom et al., 2013). La construcción de profesiones socialmente valoradas se encuentra íntimamente unida con la manera en que el poder público las gestione y las políticas que desarrolle para afrontarlas. La Comunidad de Madrid, a través de sus entidades gestoras, parecería que está permitiendo la progresiva transformación en la consideración y construcción del sector al permitir pequeñas transformaciones que sumadas en el tiempo y en cantidad generan transformaciones estructurales en el sector. Además, como señala Moreno Colom et al. (2013), tampoco existe una demanda social clara de mejores y más servicios de atención a la dependencia ni las trabajadoras del sector se encuentran en condiciones para reivindicar mayor respeto a la profesionalización de su trabajo.

\section{Conclusiones}

La Ley de Promoción de la Autonomía Personal y Atención a las Personas en Situación de Dependencia significó un enorme cambio en relación con el tratamiento de las limitaciones vitales de la población mayor. En primer lugar, reconoció el derecho de todas las personas con dificultades para realizar su vida diaria (independientemente de su edad) a acceder a los servicios públicos necesarios para mejorar su situación; y en segundo lugar, implicó el establecimiento de un modelo estatal de provisión de estos servicios, aunque delegando las competencias a las comunidades autónomas. La implantación de esta Ley, sin embargo, ha seguido una trayectoria desigual en las distintas regiones, en función de sus estructuras previas y del contexto económico y político.

En este artículo se ha llevado a cabo un análisis de la situación de la Ley de Dependencia en la Comunidad de Madrid en uno de sus variados aspectos: el servicio de atención a domicilio. La Ley proporciona, según se establece en su articulado, prestaciones diversas que intentan abarcar todas las posibles situaciones que la población dependiente pueda presentar, siendo el SAD uno de ellos. La región de Madrid se ha caracterizado por otorgar relevancia a este servicio, tanto desde antes de la aplicación de la Ley como posteriormente a ésta, cuestión que se puede observar en los datos oficiales. El índice de cobertura en la región es el más alto de todo el Estado español, aunque la intensidad en la prestación del servicio se encuentra en puestos más relegados. Existe, por tanto, una apuesta por cubrir un mayor número de personas posibles, aunque prestándoles una asistencia de tiempo limitada y haciendo que el servicio deba combinarse con otro tipo de recursos (públicos o privados).

Los últimos años, sin embargo, se han caracterizado por la diminución en la importancia del servicio y su creciente desprofesionalización. El cambio de paradigma en el SAD puede apreciarse tanto en los datos de tipo cuantitativo como en los discursos de sus protagonistas. Los datos reflejan que, tras unos primeros años de consolidación de la atención, 
ésta se ha ido reduciendo, lo cual se observa tanto en el número absoluto de personas beneficiarias como en el descenso del peso porcentual de este servicio con respecto a otras prestaciones que han aumentado de forma notable. Así, el SAD ya no representa el servicio más representativo en la región, sino que ahora es una prestación más individualizada y desprofesionalizada la que ocupa esta posición, la prestación económica por cuidados familiares. Estos cambios pueden interpretarse como una transformación en la concepción misma de los cuidados de larga duración a la población mayor, ya que la atención profesionalizada en el hogar está perdiendo peso frente a otras opciones que potencian otro tipo de cuidados. Por otra parte, el funcionamiento concreto y cotidiano del SAD también está experimentando modificaciones que reflejan una tendencia a la desprofesionalización. El establecimiento de la jornada laboral, la regulación horaria o las tareas que engloba el servicio se han ido deteriorando, de manera que las empleadas en el SAD asisten a la construcción de su profesión como una actividad informal de ayuda (similar a la consideración de un cuidador familiar o de una empleada de hogar) más que como una categoría profesional. La formación continua de las profesionales o la presencia de empresas gestoras no especializadas también se encuadran dentro de esta línea de reconfiguración.

En definitiva, la información obtenida indica una tendencia a la reconstrucción del sector del servicio de atención a domicilio como un ámbito no profesional ligado a la concepción tradicional familiar y femenina de los cuidados. Se debería, en consecuencia, presionar para que las políticas públicas implantadas por la Comunidad de Madrid no generen transformaciones estructurales en el sector que incidan en su desprofesionalización, sino que, al contrario, vayan encaminadas a proporcionar un servicio de calidad a sus usuarios que cumpla con los objetivos primigenios de la legislación. 
BÓDALO LOZANO, E. (2006): “Envejecimiento y discapacidad: una aproximación al caso español desde la perspectiva del bienestar social”, Portularia, vol. 6, nº 1, págs. 67-78.

COMUNIDAD DE MADRID (2015a): “Resolución 8/2015, del Director General de Coordinación de la Dependencia, por la que se desarrolla la disposición adicional segunda de la Orden 141/2011, de 1 de marzo, por la que se regula el catálogo de servicios y prestaciones para las personas reconocidas en situación de dependencia en grado I en la Comunidad de Madrid", Boletín Oficial de la Comunidad de Madrid, no 23, 28-1-15. [<http://www.bocm.es/ boletin/CM_Orden_BOCM/2015/01/28/BOCM20150128-21.PDF〉].

- (2015b): "Decreto 54/2015, de 21 de mayo, por el que se regula el procedimiento para reconocer la situación de dependencia y el derecho a las prestaciones del sistema para la autonomía y atención a la dependencia en la Comunidad de Madrid", Boletín Oficial de la Comunidad de Madrid, no 123, 26-5-15. [khttp://w3.bocm.es/ boletin/CM_Orden_BOCM/2015/06/24/BOCM20150624-1.PDF〉].

- (2002): “Decreto 88/2002, de 30 de mayo, por el que se regula la prestación de Ayuda a Domicilio del Sistema de Servicios Sociales de la Comunidad de Madrid", Boletín Oficial de la Comunidad de Madrid, n- 136, 10-6-02. [<http://www.bocm.es/boletin/CM_Boletin_ BOCM/20020610_B/13600.PDF〉].

DE ASÍS ROIG, R. (2007): “Reflexiones en torno a la Ley de Promoción de la Autonomía Personal y atención a las personas en situación de dependencia”, Universitas. Revista de Filosofía, Derecho y Política, nํㅜ 5, págs. 3-21.
ESPAÑA (2015): “Real Decreto 291/2015, de 17 de abril, por el que se modifica el Real Decreto 1051/2013, de 27 de diciembre, por el que se regulan las prestaciones del Sistema para la Autonomía y Atención a la Dependencia, Establecidas en la Ley 39/2006, de 14 de diciembre, de Promoción de la Autonomía Personal y Atención a las Personas en Situación de Dependencia”, Boletín Oficial del Estado, n- 104, 1-5-15. [<https://www.boe.es/boe/dias/2015/05/01/ pdfs/BOE-A-2015-4786.pdf>].

- (2013): "Real Decreto 1051/2013, de 27 de diciembre, por el que se regulan las prestaciones del Sistema para la Autonomía y Atención a la Dependencia, Establecidas en la Ley 39/2006, de 14 de diciembre, de Promoción de la Autonomía Personal y Atención a las Personas en Situación de Dependencia”, Boletín Oficial del Estado, no $313,31-12-13$ [/http://www.boe.es/boe/ dias/2013/12/31/pdfs/BOE-A-2013-13811.pdf>].

EUROFOUND (2015): Delivering Public Services: A Greater Role for the Private Sector? An Exploratory Study in Four Countries, Luxembourgo, Oficina de Publiaciones de la Unión Europea.

FANTOVA AZCOAGA, F. (2013): "Políticas públicas y profesionalización de los cuidados", Jornadas 'Los servicios de cuidado de larga duración: retos de futuro ante el bienestar cotidiano', Barcelona, octubre.

GARCÍA HERRERO, G. (2009): El servicio de ayuda a domicilio en la encrucijada. Análisis y reflexiones sobre el presente y el futuro del servicio de ayuda a domicilio, tras la implantación del Sistema de Atención a la Dependencia, Asociación Estatal de Directoras y Gerentes de Servicios Sociales.

GARCÍA MARTÍN, P. (2013): “Estudio del funcionamiento del servicios de ayuda a domicilio en 
Majadahonda", Trabajo Social Hoy, no 69, págs. 57-80.

GONZÁLEZ ORTEGA, S. (2012): La aplicación de la Ley de Dependencia en España, serie Estudios, Madrid, Consejo Económico y Social.

HOCHSCHILD, A. (2001): "Las cadenas mundiales de afecto y asistencia y la plusvalía emocional", en HUTTON, W.; y GIDDENS A., En el límite: la vida en el capitalismo global, Barcelona, Tusquets, págs. 187-208.

IMSERSO (2012): Informe 2012. Las personas mayores en España. Datos estadísticos estatales y por comunidades autónomas, Madrid, Imserso.

- (s/d) Sistema de Autonomía y Financiación a la Dependencia. Estadísticas. Datos de gestión [〈http://www.dependencia.imserso.es/ dependencia_01/documentacion/estadisticas/ est_inf/>].

INE (2011): Censo de Población y Vivienda 2011 [rhttp:// www.ine.es/censos2011_datos/cen11_datos_ inicio.htm>].

- (2009-2010): Encuesta de Empleo del Tiempo [khttp:// www.ine.es/dyngs/INEbase/es/operacion.htm $? c=$ Estadistica_C\&cid $=1254736176815$ \&menu $=$ resultados\&idp $=1254735976608>$ ].
- (s/d) Estadística del Padrón Continuo [<http://www.ine. es $/$ jaxi/menu.do?type=pcaxis $\&$ path $=/$ t2o $/$ e245/\&file=inebase $]$.

MARTíNEZ BUJÁN, R. (2014): “Los modelos territoriales de organización social del cuidado a personas mayores en los hogares", Revista Española de Investigaciones Sociológicas, nํㅜ 145 , págs. 99-126.

MINGUELA RECOVER, M. A.; y CAMACHO BALLESTA, J. A (2015): "Cuidados mixtos y cuidados informales a los mayores dependientes, ¿son complementarios o son sustitutivos?: una visión comparada entre los países del sur de Europa, Zerbitzuan, no 58 , págs. 15-25 [rhttp:// dx.doi.org/10.5569/1134-7147.58.02)].

MORENO FERNÁNDEZ, L. (2003): “Bienestar mediterráneo y 'supermujeres”, serie Documentos de Trabajo no 03-09, CSIC, Unidad de Políticas Comparadas.

MORENO COLOM, S., et al. (2013): “El trabajo de cuidados en los regímenes de bienestar: ¿más dependencia y menos profesionalidad?", IV Congreso de la Red Española de Política Social (REPS): 'Las políticas sociales entre crisis y post-crisis', Alcalá de Henares, junio. 